\title{
La politique de production cinématographique suisse : direction Sud / direction Est
}

Teresa Hoefert de Turégano

\section{CpenEdition}

\section{Journals}

Édition électronique

URL : http://journals.openedition.org/decadrages/563

DOI : $10.4000 /$ decadrages.563

ISSN : 2297-5977

Éditeur

Association Décadrages

Édition imprimée

Date de publication : 10 avril 2004

Pagination : 111-118

ISBN : 978-2-9700582-0-5

ISSN : 2235-7823

\section{Référence électronique}

Teresa Hoefert de Turégano, «La politique de production cinématographique suisse : direction Sud / direction Est », Décadrages [En ligne], 3 | 2004, mis en ligne le 29 avril 2013, consulté le 19 avril 2019. URL : http://journals.openedition.org/decadrages/563; DOI : 10.4000/decadrages.563 


\section{La politique de production cinématographique}

\section{suisse : direction Sud / direction Est}

\author{
par Teresa Hoefert de Turégano
}

La collaboration cinématographique entre la Suisse et des réalisateurs des pays du Sud et de l'Est demeure peu connue. Pourtant, la Suisse mène depuis les années 1980 une politique culturelle dans ce domaine. Les enjeux de cette politique sont d'abord socioculturels, mais aussi politiques et économiques. Cette collaboration est possible principalement grâce aux mécanismes de l'aide au développement et à la coopération des Affaires étrangères, qui peuvent être distingués des mécanismes de soutien cinématographique de l'Office fédéral de la culture (OFC). Il est bien connu que les sommes d'argent globalement disponibles en Suisse pour soutenir le cinéma sont parmi les plus basses de tous les pays européens ${ }^{\mathbf{1}}$, mais il est moins connu que les moyens de soutien pour les cinéastes de régions moins fortunées ou en difficulté dans leur propre pays sont tout aussi bas. Néanmoins, la Suisse jouit d'une certaine visibilité à l'étranger dans ce domaine.

Le plus souvent, il s'agit d'une collaboration plutôt que d'une coproduction proprement dite, car cette dernière pratique implique le partage de la propriété du négatif du film. La Suisse a signé des accords de coproduction avec l'Allemagne, l'Autriche, la Belgique, le Canada, la France et l'Italie au niveau bilatéral, et avec l'Union européene (EURIMAGES) au niveau multilatéral, ce qui rend la coproduction avec d'autres pays moins aisée. Ces accords stipulent, selon des conditions spécifiques, une égalité de traitement entre un film de "nationalité suisse» et un film coproduit, favorisant ainsi les possibilités de soutien cinématographique dans chaque pays coproducteur. S'engager dans des coproductions officielles avec des producteurs de pays dépourvus d'accords en la matière constitue alors un défi majeur, mais néanmoins envisageable $\mathbf{2}$ : il y a en effet un petit nombre de producteurs suisses qui œuvrent dans ce domaine avec plus ou moins de bonheur ${ }^{3}$.

Il y a davantage de coopération avec des cinéastes et des producteurs de pays d'Afrique, d'Asie, d'Amérique latine et de l'Europe de l'Est dans
1 En 2004, le budget fédéral alloué à l'encouragement du cinéma en Suisse se chiffre à environ 35 millions de francs, y compris pour les coproductions minoritaires. Les mesures concernant l'encouragement à la production en Suisse sont nettement inférieures à celles en vigueur dans d'autres petits pays d'Europe, tels que la Belgique, I'Irlande, l'Autriche, la Suède, le Danemark, le Portugal ou les Pays-Bas. Voir Marcel Hoehn, "Réseau européen pour les producteurs ", in Ciné-bulletin, propos recueillis par Robert Richter, n³34, août 2003, p. 10-11.

2 Citons par exemple le dernier film de Fernando Solanas, Memoria del Saqueo (2004, Suisse-France-Argentine), une coproduction suisse majoritaire produite par Pierre-Alain Meier (Thelma Films).

3 Citons à cet égard Pierre-Alain Meier, Marco Müller, Tiziana Soudani et Silvia Voser, entre autres. 
4 Sophie Delessert et Toni Linder, entretien avec l'auteur, Berne, 10 janvier 2001.

5 "Les soutiens suisses aux films d'Afrique, d'Asie et d'Amérique latine", table ronde au Festival International de Films de Fribourg (FIFF), 15 mars 2002. Invités: Jean-Pierre Bekolo (cinéaste), Sophie Delessert (DDC), Pierre-Alain Meier (producteur/réalisateur), Marco Müller (Fondazione Montecinemaverità), Ariel Rotter (cinéaste), Silvia Voser (productrice). Modératrice: Teresa Hoefert de Turégano (Université de Lausanne).

6 DDC, "Programme de promotion des films de la DDC" (Vademecum à l'intention des producteurs, des auteurs, etc.). Vademecum dfe.doc / 5 novembre 2001. un circuit parallèle à l'OFC qui tend à faciliter les processus de production. Il s'agit en premier lieu de la Direction du développement et de la coopération suisse (DDC) et de la Fondation Montecinemaverità (FMCV), ainsi que d'autres acteurs tels que trigon-film - le distributeur de films spécialisé dans ce domaine -, des festivals de films spécifiques, et des producteurs suisses indépendants qui s'engagent sur ce terrain.

L'organisation maîtresse de cette politique cinématographique est la Direction du développement et de la coopération qui fait partie du Département fédéral des affaires étrangères (DFAE), tout en gérant indépendamment son budget. Celui-ci est voté directement par le Parlement et est donc soumis à une approbation plus directe de la part du public suisse. A partir de 1981, Toni Linder entre en fonction à la DDC et tente de créer des actions en faveur du cinéma qui demeurent toutefois exceptionnelles. Ce n'est qu'en 1988 qu'une véritable politique de soutien est instaurée. La DDC intervient alors dans la production et la distribution des films. Aujourd'hui, cette aide pour le soutien cinématographique qui s'élève à environ 2 millions de francs est allouée dans le cadre d'un budget de près de 7 millions de francs 4 . Sophie Delessert, actuellement en charge de ce dossier, souligne que "le soutien à la production doit inciter à une prise de conscience des impératifs de la politique du développement. [...] Il n'est pas destiné à la promotion de la production de films dans le tiers-monde» $\mathbf{5}$. Ce soutien n'est donc pas conçu comme une aide au développement traditionnel, mais comme une politique d'éducation et de sensibilisation des habitants de la Suisse aux différentes cultures dans le monde. Relevons la franchise de cette politique qui ne cache pas la primauté de ses propres intérêts, phénomène qui constitue généralement la face dissimulée de l'aide au développement. En même temps, cette politique permet un réel soutien à certains cinéastes de pays d'Afrique, d'Asie, d'Amérique latine et d'Europe de l'Est sans imposer des conditions draconiennes.

$\mathrm{Au}$ niveau de la production, la DDC a d'abord alloué une aide au documentaire et par la suite à la fiction. Depuis 1992, l'aide de la DDC pour les films de fiction est attribuée par la Fondation Montecinemaverità, tandis que les documentaires et les courts métrages continuent d'être soutenus directement par la DDC6. Il faut souligner la qualité de leur soutien au documentaire, mais notons que les conditions d'octroi d'une aide se durcissent de plus en plus: aujourd'hui, pour qu'un documentaire soit soutenu par cette institution, il faut déjà pouvoir compter sur la participation d'une chaîne de télévision. L'aide à la distribution de la DDC s'achemine par le biais d'un soutien à trigon-film, une maison suisse de distribution de films d'Afrique, d'Asie 
et d'Amérique latine. La DDC soutient également divers événements et des programmations ponctuelles de films, ainsi que plusieurs festivals, tels que le Festival International de Films de Fribourg, le Festival Média Nord/Sud, Black Movie, Cinemafrica ${ }^{7}$. Ajoutons que depuis 2003, elle fournit une aide substantielle au Festival International de Films de Locarno pour une programmation s'ouvrant aux cinémas du Sud8. L'aide de la DDC n'est pas uniquement destinée à augmenter la visibilité de différentes cultures en Suisse: en marge, elle soutient aussi certains projets et actions sur place dans les pays en question. Ainsi, l'Ecole Internationale de Cinéma et de Télévision (EICTV) de San Antonio de los Baños à Cuba reçoit un soutien matériel et en équipements, ainsi que des bourses pour les étudiants étrangers et des échanges de professeurs.

Si un acteur de cette politique cinématographique suisse a pu acquérir une certaine notoriété à l'étranger, principalement dans les circuits internationaux du cinéma indépendant, il s'agit bien de la Fondation Montecinemaverità (FMCV). Celle-ci a été créée en 1992 à Locarno, à travers le soutien de la Direction du développement et de la coopération (DDC) et d'United Colours Communication SA (UCC), société pour la communication du Groupe Benetton, dans le but de promouvoir et de consolider le développement d'un cinéma indépendant dans les pays du Sud du monde et de l'Est de l'Europe ${ }^{9}$. En tant que directeur du Festival International de films de Locarno à l'époque, Marco Müller lance cette fondation dont il devient le dirigeant. Il faut ainsi ajouter la FMCV à une petite liste de fondations cinématographiques dans le monde (par exemple, Hubert Bals Fund, Fonds Sud, Sundance, etc.) qui constituent un véritable moteur dans la cinématographie indépendante de l'Asie, d'Afrique, d'Amérique latine et d'Europe de l'Est.

Les sommes allouées par la FMCV et la DDC sont surtout de nature incitative; néanmoins, elles peuvent jouer un rôle absolument crucial dans la possibilité d'existence d'un film. Le financement varie globalement entre 20000 et 50000 francs; en moyenne, six à huit films sont soutenus par année. La FMCV recevait de la DDC 150000 francs par année pour les films du Sud jusqu'en 1997, somme qui a par la suite doublé (300000 francs) pour inclure une aide aux films de l'Est 10. Aux principaux partenaires de la FMCV que sont la DDC et l'UCC se sont au fur et à mesure ajoutés Swisscom, SSG-SRG qui transite par la TSI, la Ville de Lugano et le Canton de Tessin. La FMCV disposait, pour le soutien direct aux projets, d'une somme d'environ 480000 francs en 1991, 470000 en 2002 et 350000 en 2003. Le financement de la fondation repose presque paritairement sur des fonds publics et privés et, selon M. Müller, les partenaires privés n’interviennent pas dans la sélection
7 Brugger, Hanser und Partner AG (Ernst Brugger, Rolf Keller, Guido Calvelti), "Evaluation DEZA-Filmförderung" (Schlussbericht zu Handen Herrn T. Linder, DEZA), Zürich, 7 février 2000.

8 II s'agit de 550000 francs $\times 3$ ans. Sophie Delessert, conversation téléphonique avec l'auteur, 16 février 2004.

9 Fondation Montecinemaverità, Ascona, Active support to motion picture production in the Southern hemisphere and Eastern Europe - Regulations (based on art. 2 and 8 of the statutes of the Montecinemaverità Foundation, joined to the founding act of $15^{\text {th }}$ October 1992).

10 Fondation Montecinemaverità, "Détail des soutiens aux projets de longs métrages", 2000. 
11 "Les soutiens suisses aux films d'Afrique, d'Asie et d'Amérique latine", table ronde du Festival International de Films de Fribourg (FIFF), 15 mars 2002.

12 Sophie Delessert et Toni Linder, entretien avec l'auteur, Berne, 10 janvier 2001.

13 DDC, "Programme de promotion des films de la DDC" (Vademecum à l'intention des producteurs, des auteurs, etc.). Vademecum dfe.doc / 5 novembre 2001.

14 Sophie Delessert et Toni Linder, entretien avec l'auteur, Berne, 10 janvier 2001.

15 En vue d'obtenir l'argent fédéral de l'OFC pour une coproduction suisse, il faut en général (sauf si un accord de coproduction stipule des termes spécifiques) disposer d'au moins $50 \%$ du financement global, et réaliser le film en Suisse, avec des Suisses, en dépensant l'argent en Suisse ou pour des Suisses. Quand il s'agit de pays avec lesquels la Suisse n'a pas passé d'accord officiel, les conditions d'obtention de l'appellation suisse sont décidées au cas par cas.

16 Laurence Hoffmann (coordinatrice FMCV), entretien avec l'auteur, Soleure, 22 janvier 2004. des films 11. A titre de comparaison avec d'autres fondations européennes, on peut avancer les chiffres suivants: le Fonds Sud en France dispose d'environ 2 millions d'euros (près de 3 millions de nos francs), le Fond Hubert Bals au Pays-Bas de 935000 euros (environ 1 million 400000 francs), le nouveau fond de la Berlinale en Allemagne de 1 million d'euros (1,5 million de francs), le Göteborg Film Fund en Suède de 300000 euros (450000 francs), et le Fond Novo en Belgique d'environ 600000 euros (900000 francs).

Dans le cadre restreint de la production et de la coproduction internationale indépendantes, de telles fondations peuvent devenir des marques de "distinction" et apporter à un film promu des bénéfices audelà de l'aspect financier. En soutenant un projet, elles peuvent encourager la participation d'autres subventionneurs éventuels et constituer une porte d'entrée en regard des programmateurs de grands festivals, des distributeurs, des télévisions, etc. C'est dire que les fondations jouent un rôle non négligeable. Les stratégies politiques et économiques qui visent à se distinguer des autres au travers d'une garantie de qualité correspondent à une ligne de conduite souvent employée par les petits pays et plus particulièrement par la Suisse dans des domaines extra-culturels. Alors, pourquoi ne pas l'appliquer davantage au domaine culturel?

Les critères de sélection de la FMCV sont comparables dans leur esprit à ceux de la DDC12, encore que les spécifications de la DDC soient plus pointilleuses. Dans les deux cas, le choix porte sur des projets d'un «haut niveau qualitatif» dans leur forme et leur contenu cinématographiques, mais la DDC fait mention d'autres préférences quelque peu équivoques. Par exemple, elle accorde son soutien à des films «qui traitent des aspects de la problématique du développement; qui sont le plus authentique possible et/ou qui sont l'expression d'une identité culturelle»13. A propos de ces préférences, S. Delessert reconnaît que "ces termes sont en effet discutables; ce qu'ils veulent dire, c'est que le film doit montrer un autre regard, une autre culture, une nouvelle manière de voir quelque chose» $\mathbf{1 4}$. Malgré ces critères, et contrairement au système de soutien de l'OFC, il n'y pas d'obligation de dépenser l'argent en Suisse $\mathbf{1 5}$; cependant, il est recommandé de bénéficier d'une coproduction suisse et de figurer sur la liste des pays prioritaires dans le programme d'aide et de coopération suisse. La FMCV pour sa part choisit «des projets qui sont pertinents par rapport au contexte social du pays producteur, mais aussi des projets qui font sens dans le contexte du marché européen » $\mathbf{1 6}$. Sur ce dernier point, la fondation répond aux exigences de la DDC consistant à promouvoir la "diversité culturelle» en Suisse. 
Curieusement, depuis la fin décembre 2002, la FMCV existe sans directeur, le Comité exécutif n'ayant pas nommé de successeur à la place de Marco Müller. Cette absence indique qu'il y a une réflexion autour de la structure actuelle de la politique cinématographique suisse dans ce domaine, et une restructuration en cours ${ }^{\mathbf{1 7}}$. La diminution récente du budget de la fondation et, avec le départ de M. Müller, le retrait de Benetton semblent corroborer cette hypothèse. L'avenir de la FMCV paraît aujourd'hui précaire; il est difficile de comprendre la logique politique qui motive ces manœuvres, car si des critiques peuvent certes être adressées à de telles fondations, elles ne justifient pas pour autant leur dissolution.

Etant donné le poids accordé à la diversité culturelle dans cette politique cinématographique, il semble important de déterminer si, et comment, les films se situent dans le discours de la diversité. Les films issus de ces structures sont-ils simplement des instances d'un exotisme cinématographique pour le spectateur occidental? Nul ne doute de l'utilité de présenter une plus grande variété d'images en Suisse. Mais, il faut le souligner, un changement quantitatif n'est pas suffisant en soi. Nous devons aussi nous interroger sur la nature de ces représentations: en quoi nos exigences et pratiques socio-culturelles, politiques, et même économiques restreignent-elles et conditionnent-elles nos critères d'évaluation et de valorisation de la cinématographie de certains pays?

Dans son introduction à la programmation 2003 du Festival de Locarno, Irene Bignardi (directrice du festival) a souligné comme nouveauté une "ouverture au cinéma dit du monde ou du Sud». Parmi ces "cinématographies en latence, qui n’arrivent pas à réaliser leur potentiel pour des raisons politiques ou économiques, Cuba était un choix évident: ce pays, qui produisait une vingtaine de films par an, n'en sort désormais plus que quatre ou cinq et avec beaucoup de difficultés, alors que le talent, les idées et les structures existent»18. Ajoutons encore que ces quatre ou cinq films sont en général des coproductions avec des pays européens, c'est-à-dire des films qui bénéficient par exemple de l'argent des fondations déjà mentionnées et d'autres structures similaires. Dans beaucoup de cas, ces fondations sont liées à des festivals de films, ce qui constitue pour eux une source d'approvisionnement. En effet, les films soutenus par la FMCV sont souvent sélectionnés pour la programmation de Locarno et de Fribourg, et parfois distribués par trigon-film $\mathbf{1 9}$.

Trigon-film est une fondation qui n'est pas strictement orientée vers le profit; elle a comme but «de battre en brèche les préjugés culturels» et «d'ouvrir les écrans quelques fois par an sur des espaces moins familiers que ceux de l'Occident ${ }^{20}$. Pour certains, trigon-film connote un
17 Claude Girardin, "Nouvelle étape pour la Fondation Montecinemaverità ", in Ciné-bulletin, $n^{\circ} 334$, août 2003, p. 14-16.
18 "Le nouveau Locarno d'Irene Bignardi ", propos recueillis par Mathieu Loewer, in Ciné-bulletin, $\mathrm{n}^{\circ} 334$, août 2003, p. 4.

19 Fondazione Montecinemaverità, "Participation aux festivals, reconnaissances obtenues, diffusion", 2001.

20 www.trigon-film.ch 
21 Pierre Leprohon, L'Exotisme et le Cinéma, Editions J. Susse, Paris, 1945, p. 200.

22 Frances Affergan, Exotisme et Alterité Essai sur les fondements d'une critique de l'anthropologie, PUF, Paris, 1987, p. 17.

23 Walter Ruggle, "Le monde sans cesse redécouvert", Le tour du monde en 90 films, trigonfilm, catalogue 2001-2002, p. 7. "cinéma du monde», un peu exotique, et dont les titres inscrits au catalogue présenteraient même certaines affinités esthétiques. Pourtant, les films de trigon sont en fait plus divers qu'une certaine allure d'exotisme de l'institution ne le laisse entendre. Il n'en demeure pas moins vrai qu'on constate une disparité de la qualité des films qui constituent leur catalogue; évidemment, il faut mettre ces inégalités au compte de l'offre réduite dont dispose le distributeur, les cinéastes préférant dans certains cas bénéficier d'une diffusion plus large.

En tant qu'institution, trigon-film emblématise la complexité qu'il y a à gérer une politique culturelle dans une configuration hiérarchique et étatique très inégale. On observe un embarras entre une volonté de promouvoir l'existence de cinématographies qui fonctionnent sur une autre logique que celle du marché et entre les restrictions et la ghettoïsation qu'une telle politique peut générer. Que ces cultures aient été historiquement associées à un exotisme dans le savoir occidental n'arrange pas l'affaire. Les cinéastes ne sont d'ailleurs pas dupes de ces enjeux: comment éviter un discours exotique d'un côté et comment gérer un exotisme répandu et pour ainsi dire requis de l'autre côté?

En 1945 Pierre Leprohon écrivait: "Le cinéma exotique est d'autant plus intéressant qu'il est moins intentionnel [...] c'est que leurs réalisateurs ne s'inquiétèrent pas de plaire aux spectateurs de Paris ou de San Francisco, mais d'être conformes aux traditions de leur pays» 21. L'époque n'est plus la même mais la question de l'intentionnalité reste un enjeu, car il s'agit d'un art qui s'actualise à travers le spectateur. L'exotisme fonctionne, entre autres, par une juxtaposition entre, d'une part, une réalité banale et l'absence de mystère "d'ici» et, d'autre part, la lumière, la couleur, et la vie d'un "ailleurs» 22 . C'est précisément ce genre de discours qui ressort dans l'introduction du catalogue de trigonfilm: "Direction sud, où le soleil atteint son zénith, et est, où il se lève chaque matin, les volets de cette fenêtre restent bien souvent clos, ce qui nous prive du plaisir d'être réveillés par la lumière de l'aurore et de ressentir la chaleur du soleil de midi »23. Bien évidemment, cette tension qui se noue autour de l'exotisme constitue seulement l'une des configurations caractérisant le champ de cette politique culturelle. L'exemple de la nouvelle génération de cinéastes argentins montre bien que, pour la plupart d'entre eux, les enjeux se situent ailleurs, dans un cinéma intimiste, social, axé sur une expérience subjective de la société, et qui interroge l'identité au pluriel, en ne se souciant guère de la couleur ou de la chaleur du soleil.

Le terme "cinéma du monde» reste ainsi problématique, de par ses liens avec le terme «musique du monde» qui a des connotations péjoratives. 
Ceci constitue l'un des obstacles auxquels certains cinéastes qui circulent dans ce réseau cinématographique doivent faire face. Ainsi, pour bénéficier d'un financement, les films doivent la plupart du temps renvoyer à des questions d'authenticité et d'identité culturelle, comme si le concept de l'identité culturelle était une entité statique qui pouvait se décliner suivant un certain nombre de traits établis. Traduire en ces termes la recherche de l'Autre, d'une identité culturelle ou bien nationale, risque d'être réducteur, plus que tout autre chose.

Plus récemment, Martial Knaebel, directeur artistique du Festival International de Films de Fribourg (FIFF), spécialisé dans les cinémas du «Sud», et représentant de la DDC au comité d'experts de la FMCV, a expliqué comment le FIFF conçoit sa programmation, en proposant une notion du Sud plus élargie: "Cette notion devient plus sociologique que géographique. Nous explorons l'idée de "Sud sociologique», mais nous avons aussi la volonté de défendre la notion de diversité parce qu'il existe un problème de standardisation culturelle amené par la globalisation...»24. Laissons de côté le débat entre le sociologique et le géographique pour revenir sur la question de la diversité opposée à la standardisation culturelle.

Il ne s'agit pas en réalité d'une opposition stricte entre diversité et homogénéisation, car les deux mécanismes se nourrissent l'un l'autre 25. Comme le soulève Nestor Garcia Canclini, "les nations et les ethnies continuent d'exister. Le problème principal ne semble pas être le risque de leur effacement par la mondialisation mais plutôt de comprendre comment les identités ethniques, régionales et nationales se reconstituent»26. Dans le domaine cinématographique, une réaction courante face aux dangers de l'homogénéisation consiste à se retrancher sur les conceptions et les traditions de l'identité les moins complexes, voire réductrices, et qui tendent à la réification. Au sein même du discours sur la diversité culturelle, il est primordial d'observer comment les uns et les autres s'approprient ce discours.

C'est l'un des risques auxquels doit faire face trigon-film, tout comme d'autres institutions. Malgré son utilité pour la promotion de films qui ne parviennent pas à trouver de distributeurs, cette fondation se base sur un discours qui tend vers un certain exotisme de l'Autre, perpétuant ainsi une forme de ghettoïsation. Cependant, les activités de trigon-film me paraissent indispensables, notamment en ce qui concerne certains objectifs à long terme. Ce genre d'institution familiarise le public avec des cinématographies peu connues, ouvrant ainsi la voie à des pratiques filmiques qui n'ont plus besoin d'exhiber leur "authenticité », de s'intéresser nécessairement à «l’identité culturelle» et d'aborder
24 Cité par Mathieu Loewer et Françoise Deriaz, "Festival de Fribourg au-delà du Sud", in Cinébulletin, $n^{\circ} 341$, mars 2004, p. 6.

25 Voir, par exemple, Stuart Hall, "The Local and the Global: Globalization and Ethnicity" et "Old and New Identities, Old and New Ethnicities", Culture, Globalisation and the WorldSystem, Anthony D. King (éd.), University of Minnesota Press, Minneapolis, 1997.

26 Nestor García Canclini, "Will there be Latin American Cinema in the Year 2000? Visual Culture in a Postnational Era", Framing Latin American Cinema-Contemporary Critical Perspectives, Ann Marie Stock (éd.), University of Minnesota Press, Minneapolis/London, 1997, p. 256. 
les "problèmes de développement». Avec le temps, les coproductions peuvent se détacher d'un certain exotisme culturel et se rattacher aux créneaux dits indépendants. A cet égard, il faut également prendre en compte les principales institutions actives dans cette politique cinématographique. En fin de compte, c'est avec ces dernières que la Suisse a véritablement commencé à développer une visibilité internationale, et notamment à travers la Fondation Montecinemaverità qui a acquis une notoriété indéniable dans ce domaine. 